\title{
Sol-gel derived gold nanoparticles biosensing platform for Escherichia coli detection
}

\author{
J. Redondo-Marugan ${ }^{1}$, M.D. Petit-Dominguez ${ }^{1}$, E. Casero ${ }^{1}$, L. Vázquez ${ }^{2}$, T. García ${ }^{1}$, A.M. \\ Parra-Alfambra $^{1}$, E. Lorenzo ${ }^{1} *$
}

${ }^{1}$ Departamento de Química Analítica y Análisis Instrumental. Facultad de Ciencias. C/ Francisco Tomás y Valiente $N^{o} 7$.

${ }^{2}$ Instituto de Ciencia de Materiales de Madrid (CSIC). C/ Sor Juana Inés de la Cruz $N^{o} 3$.

Campus de Excelencia de la Universidad Autónoma de Madrid. 28049 Madrid. Spain.

*e-mail: mdolores.petit@uam.es; encarnacion.lorenzo@uam.es

\begin{abstract}
A three dimensional polymeric network obtained by the sol-gel process was applied to develop disposable, sensitive, selective and stable electrochemical biosensors for detection and quantification of Escherichia coli. This biosensor is based on the integration of a thiolated capture probe sequence from Escherichia coli and gold nanoparticles (AuNPs) into a sol-gel 3D polymeric network derived from (3-mercaptopropyl)-trimethoxysilane (MPTS), formed onto a screen-printed gold electrode surface. MPTS presents the advantage of forming a 3D polymeric network containing a large number of thiol tail groups distributed throughout its structure that enable both its anchoring onto gold surfaces and the AuNPs incorporation. Moreover, this matrix allows the incorporation of a high amount of capture probe and provides a biocompatible environment that preserves its original recognition capability after its immobilization, which is expected to improve the sensitivity of the final biosensing device. The hybridization event is detected using the ruthenium complex, $\left[\mathrm{Ru}\left(\mathrm{NH}_{3}\right)_{5} \mathbf{L}\right]^{2+}$, where $\mathbf{L}$ is the ligand [3-(2-phenanthren9-yl-vinyl)-pyridine], as redox indicator, which interacts preferentially with ds-DNA. With the use of this approach, complementary target sequences of Escherichia coli can be quantified over the range of 21 to 400 pmoles with a detection limit of 6.3 pmoles and good reproducibility $(\mathrm{RSD}=4.3 \%, \mathrm{n}=4, \mathrm{C}=10 \mu \mathrm{M})$. In addition, this approach is very selective allowing the detection, without the need of a hybridization suppressor, of a single mismatch. It is also quite stable under storage conditions, giving response at least for 25 days.
\end{abstract}

Keywords: Sol-gel network, DNA biosensor, Gold nanoparticles, Ruthenium complex, Electrochemical detection, Escherichia coli. 


\section{Introduction}

The need of simple portable devices for DNA diagnostics has resulted in considerable research efforts into DNA hybridization biosensors developing. The basic element of a DNA sensor is a single strand oligonucleotide (ss-DNA) probe, immobilized on the transducer surface. The subsequent hybridization with complementary ss-DNA sequences (target strand) has been detected by various analytical techniques based on gravimetric [1,2], radioisotope or fluorescence labelling [3,4], surface plasmon resonance [5] or quartz crystal microbalance [6]. However, most of them present important drawbacks, such as the need of expensive equipment or time-consuming data interpretations. Nowadays, great efforts are being devoted to use electrochemical transducers in the DNA biosensing device [7-9], since they present several advantages such as a low-cost instrumentation and a rapid and simple methodology, while at the same time they provide a high selectivity, inherent to the recognition element, as well as a good sensitivity supplied by the electrochemical methods.

The immobilization of single stranded DNA onto a transducer is found to be the most crucial part in the development of the biosensor due to its influence on the sensitivity, stability and accuracy of the device. In this sense, the employment of a polymeric network generated by the sol-gel technology has been widely used as an adequate matrix in which several compounds, including biological material, can be encapsulated. This matrix allows not only the encapsulation of high amount of single stranded DNA but also provides a biocompatible environment that preserves its recognition capability [7,10-14]. Moreover, the sol-gel network is simple to prepare and presents important properties, mainly its chemical inertness, high stability, physical rigidity, tuneable properties and negligible swelling in aqueous and non-aqueous solution [15-19]. Different precursors have been reported in the literature for the sol-gel network preparation. The most employed for DNA and enzyme-based biosensors have been oxysilanes, such as methyltrimethosysilane, tetraethoxysilane, tetramethoxysilane, 3-amino-propyltriethosysilane, (3-mercaptopropyl)-trimethoxysilane and 3-glycidosypropyl-trimethoxysilane [11,12,15,16,1925] and metal oxides such as $\mathrm{TiO}_{2}, \mathrm{CeO}_{2}, \mathrm{SnO}_{2}, \mathrm{ZrO}_{2}, \mathrm{NiO}$ and $\mathrm{ZnO}[10,13,14,26-28]$.

On the other hand, nanomaterials have been used in bioanalytical chemistry as a strategy to improve the analytical properties of the resulting sensor devices. Particularly, gold nanoparticles (AuNPs) have been used in a wide range of applications due to their chemical stability and physicochemical properties, such as large surface area and excellent biocompatibility $[15,29,30]$. 
In particular, recent advances have been made to improve the charge transport in bio sol-gels, such as the addition of metal nanoparticles or carbon nanotubes [10,13,14,31].

In the present work, we have designed a strategy to prepare a new DNA electrochemical sensor. It is based on the immobilization of single stranded DNA (a thiolated capture probe from the bacterium Escherichia coli was chosen as model) into a three-dimensional polymeric network generated from (3-mercaptopropyl)-trimethoxysilane (MPTS) onto the surface of screen printed gold electrodes. MPTS is known to form a 3D structure plenty of thiol tail groups, with a strong affinity to gold, distributed throughout the network. MPTS is able not only to chemisorb onto gold surfaces but also to conveniently link nanosized Au particles [32,33]. This organicinorganic hybrid material was designed with the aim of benefiting from their different components: (i) a three-dimensional polymeric network that provides a biocompatible and stable environment for single strand DNA protection, (ii) gold nanoparticles contributing to facilitate both the immobilization of the thiolated capture probe and the electron transfer kinetics by acting as tiny conduction centers, and (iii) recognition element, single stranded DNA, offering a high selectivity. After exposition of this biosensing platform to a solution containing the target sequence, the hybridization event was detected using differential pulse voltammetry (DPV) from changes in the electroactivity of the redox indicator pentaamin ruthenium [3-(2-phenanthren-9yl-vinyl)-pyridine] complex, previously incorporated into ds-DNA layer formed after hybridization. This compound is capable of binding to double-stranded DNA more efficiently than to single-stranded DNA in a mode that is fundamentally intercalative due to the presence of an aromatic group in the ligand moiety [8,34-36], allowing the detection not only of a complementary Escherichia coli sequence but also the presence of a mismatched sequence.

\section{Materials and methods}

\section{Chemicals and solutions}

Synthetic oligonucleotides used in this work were 25-mer sequences from the bacterium Escherichia coli and Listeria. We employed five different sequences of DNA:

Capture probe (EclSH): 5'-HS( $\left.\mathrm{CH}_{2}\right)_{6}$-CAGGATATGTGGCGGATGAGCGGCA， sequence modified at the 5 '-end with a hexaalkythiol moiety.

Complementary target $(E c 2 c): 5$ '-TGCCGCTCATCCGCCACATATCCTG

Complementary target with a single mismatched (Ec2m): 5'-TGCCGCTCATCCACCACATATCCTG Non complementary target (Ec2nc): 5'-GATTATGACGAATAAGTGCGCAAGT 


\section{Listeria target: 5'-AGTGAGTGCGGTTAGACCTGCTAGG}

These custom-made sequences were purchased from Sigma-Aldrich and were dissolved in $0.1 \mathrm{M} \mathrm{pH}=7$ sodium phosphate buffer to obtain $100 \mu \mathrm{M}$ stock solutions.

The pentaamin ruthenium [3-(2-phenanthren-9-yl-vinyl)-pyridine] complex, $\left[\mathrm{Ru}\left(\mathrm{NH}_{3}\right)_{5} \mathrm{~L}\right]^{2+}$, was prepared by mixing equimolecular amounts of $\left[\mathrm{Ru}\left(\mathrm{NH}_{3}\right)_{5}\left(\mathrm{H}_{2} \mathrm{O}\right)\right]\left(\mathrm{PF}_{6}\right)_{2}$ and the ligand $\mathrm{L}$, (3(2-phenanthren-9-yl-vinyl)-pyridine), by ultrasound bath for 30 minutes at room temperature. Stock solutions of $\left[\mathrm{Ru}\left(\mathrm{NH}_{3}\right)_{5}\left(\mathrm{H}_{2} \mathrm{O}\right)\right]\left(\mathrm{PF}_{6}\right)_{2}$ and $\mathrm{L}$ were prepared just prior to use by dissolving the solids in $\mathrm{KNO}_{3} 5 \mathrm{mM}$ and $\mathrm{DMF}$ respectively. The ligand (L) was synthesized according to a procedure previously reported [8] and the complex $\left[\mathrm{Ru}\left(\mathrm{NH}_{3}\right)_{5}\left(\mathrm{H}_{2} \mathrm{O}\right)\right]^{2+}$ was synthesized from $\left[\mathrm{Ru}\left(\mathrm{NH}_{3}\right) \mathrm{Cl}\right]^{2+}$ by standard methods [37]. (3-Mercaptopropyl)trimethoxysilane, MPTS, was provided from Aldrich and colloidal gold nanoparticles $(20 \mathrm{~nm}$ particle size) were purchased from Sigma Chemical Co. All chemicals used for the preparation of stock and standard solutions were of analytical grade and ultra-pure water was obtained using a Milli-RO Milli-Q Plus (18.2 $\mathrm{M} \Omega \cdot \mathrm{cm}$, Millipore Water system). All solutions were prepared just prior to use and all the experiments were carried out at room temperature.

\section{Apparatus and instrumentation}

Differential pulse voltammetric (DPV) and cyclic voltammetric (CV) measurements were performed with a $\mu$-Stat 100 portable electrochemical analyzer (Dropsens, Spain) connected to a computer and to an electrochemical cell adapted to screen-printed electrodes. 220-AT gold screen-printed electrodes were purchased from Dropsens (Spain). They include a three electrode configuration printed on the same strip. The dimensions of the strips are $3.4 \mathrm{~cm} \mathrm{x} 1.0 \mathrm{~cm} \times 0.05$ $\mathrm{cm}$ (length $\mathrm{x}$ width $\mathrm{x}$ height). They present a $4 \mathrm{~mm}$ diameter disk gold working electrode and counter electrode, both printed on alumina substrate using heat curing composite inks, and a silver pseudo-reference electrode. An insulated layer serves to delimit the working area and electric contacts. Ink formulation and production characteristics of commercial screen-printed electrodes are regarded by the manufacturers as proprietary information. A specific connector allows the connection of the electrochemical strip to the electrochemical analyzer.

Atomic Force Microscopy (AFM) images were obtained with a Nanoscope IIIa equipment (Veeco, USA) operating in dynamic mode in air conditions. Silicon cantilevers (Veeco, USA) with a nominal radius of $8 \mathrm{~nm}$ and force constant in the $1-5 \mathrm{~N} / \mathrm{m}$ range, were employed. The 
Scanning Electron Microscopy (SEM) images were obtained with a NOVA NANOSEM 230 equipment (FEI) operating with a VCD (low voltage-high contrast) detector that allows us to simultaneously detect the secondary and backscattered electron signals.

AFM and SEM measurements were performed using gold supports that consisted of glass substrates $(1.1 \mathrm{~cm} \times 1.1 \mathrm{~cm})$ covered with a chromium adhesion layer $(1-4 \mathrm{~nm}$ thickness $)$ in which a gold layer (200-300 nm thickness) was deposited (Arrandee Co. Werther, Germany). In both cases gold surfaces were previously annealed for $2 \mathrm{~min}$ in a gas flame in order to obtain $\mathrm{Au}$ (111) terraces. After the annealing procedure, the surface morphology of the gold substrates imaged by AFM displayed a granular morphology with lateral sizes of a few microns separated by relatively deep grooves. However, the surface of these grains is quite smooth and flat, below the nm level. On top of the as-prepared gold surfaces were deposited for characterization the different samples (see below).

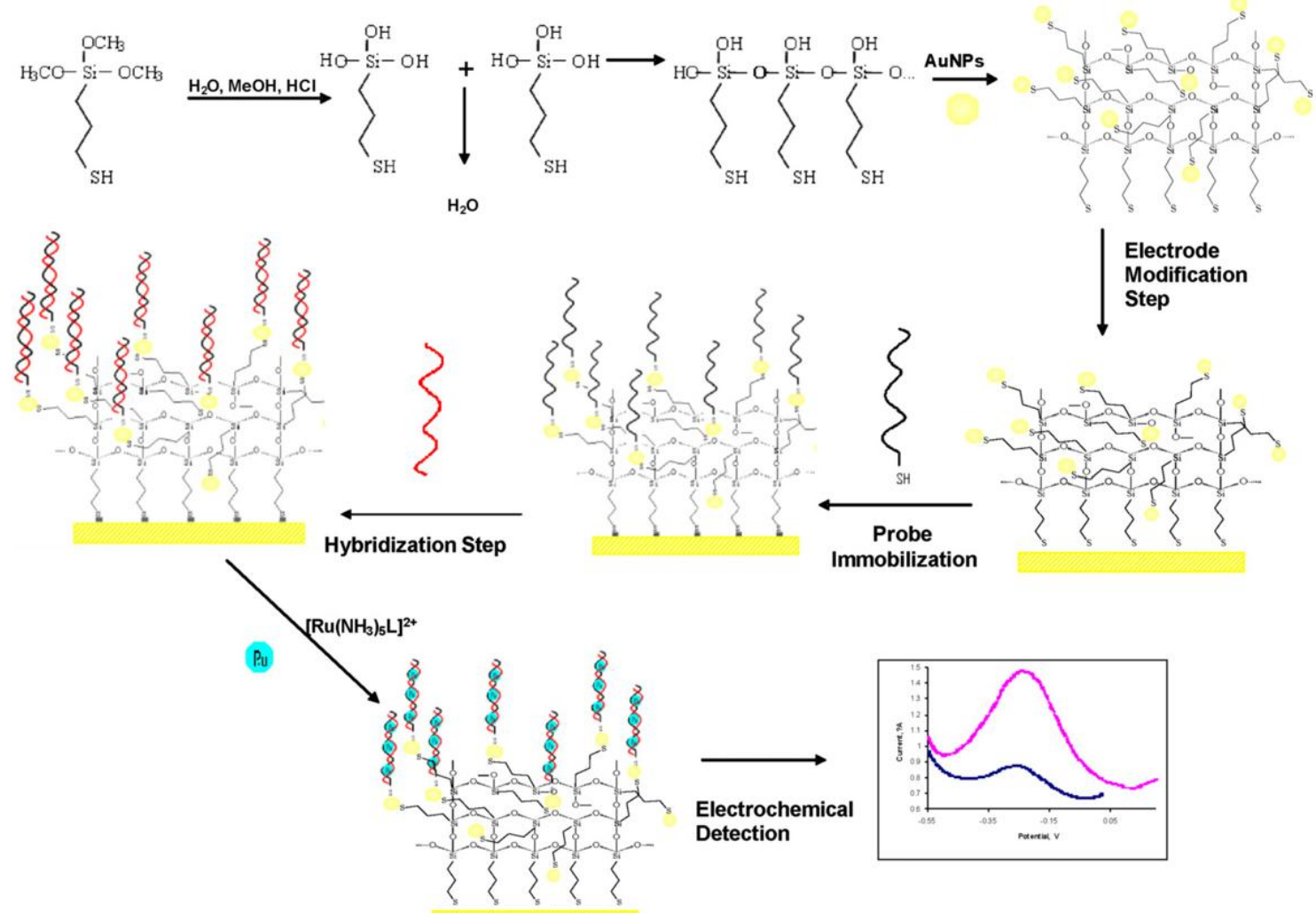

Scheme 1. Schematic representation of the different steps involved in the preparation of the modified electrode. 


\section{Procedures}

\section{Electrode activation}

The gold surfaces of working electrodes were conditioned to obtain a proper baseline and stable response, by applying ten cycles of $\mathrm{CV}$ between $-0.35 \mathrm{~V}$ and $1.2 \mathrm{~V}$ in $0.1 \mathrm{M} \mathrm{H}_{2} \mathrm{SO}_{4}$, at a scan rate of $100 \mathrm{mV} \mathrm{s}^{-1}$.

\section{Sol-gel solution preparation}

A sol-gel solution was prepared by mixing $400 \mu \mathrm{L}$ of MPTS with $1200 \mu \mathrm{L}$ of methanol, 900 $\mu \mathrm{L}$ of water and $400 \mu \mathrm{L}$ of $0.1 \mathrm{M} \mathrm{HCl}$. After mixing by ultrasonic agitation, $300 \mu \mathrm{L}$ of gold nanoparticles colloid solution was added. Au nanoparticles chemisorb onto the thiol groups of the MPTS (Scheme 1). Finally, the mixture was stored for two hours at room temperature. A similar sol-gel solution, but without AuNPs, was prepared replacing gold nanoparticles colloid solution by $300 \mu \mathrm{L}$ of water.

\section{Biosensor preparation}

The preparation of the Ec1SH/AuNPs/MPTS/Au biosensor was carried out by placing 10 $\mu \mathrm{L}$ of the MPTS sol-gel solution containing AuNPs, prepared as described above, on the gold electrode for fifteen minutes. MPTS chemisorbs on the Au electrode forming a 3D silicate network. The resulting AuNPs/MPTS/Au electrode was thoroughly rinsed with water to remove the physically adsorbed MPTS sol. Afterwards, $10 \mu \mathrm{L}$ of different concentrations of HS-ssDNA (Ec1SH) solution was placed onto the surface of AuNPs/MPTS/Au electrode (see Scheme 1). In the optimal conditions, modified electrodes were obtained by transferring $10 \mu \mathrm{L}$ of $100 \mu \mathrm{M}$ HSssDNA (Ec1SH). Then, the resulting modified electrode was air-dried and rinsed with deionized water to eliminate the non-specifically adsorbed Ec1SH. The platform denoted as Ec1SH/MPTS/Au was carried out in the same way, but by placing $10 \mu \mathrm{L}$ of the MPTS sol-gel solution (without AuNPs) on the gold electrode. The biosensor denoted as Ec1SH/Au was constructed by placing $10 \mu \mathrm{L}$ of $100 \mu \mathrm{M}$ HS-ssDNA (Ec1SH) solution onto a screen-printed gold electrode. Then, the resulting modified electrode was air-dried and rinsed with deionized water.

\section{Hybridization over Ec1SH-modified electrode and electrochemical detection}

Gold screen-printed electrodes modified with thiolated DNA probe were incubated in solutions containing $10 \mu \mathrm{L}$ of different concentrations of fully complementary (Ec2c), 
mismatched (Ec2m) or non-complementary (Ec2nc) E. Coli ss-DNA sequences. For the interference study, the incubation was carried out in presence of a mixture of fully complementary E. Coli and Listeria sequences (Ec2c+Lis). These assemblies were kept at $40^{\circ} \mathrm{C}$ for 1 hour to achieve hybridization. Electrodes were then rinsed with deionized water to remove non-specifically bound oligonucleotides. Afterwards, $10 \mu \mathrm{L}$ of $100 \mu \mathrm{M}\left[\mathrm{Ru}\left(\mathrm{NH}_{3}\right)_{5} \mathrm{~L}\right]^{2+}$ (in a 5 $\mathrm{mM} \mathrm{KNO} 3$ solution) were placed on their surface, cycling the potential from -0.5 to $0.1 \mathrm{~V}$ at 100 $\mathrm{mV} \mathrm{s}^{-1} 150$ times. Afterwards, electrodes were rinsed with deionized water and DPVs were immediately recorded in $1 \mathrm{M} \mathrm{KNO}_{3}$ as supporting electrolyte. The instrumental conditions employed in DPV are: $50 \mathrm{mV}$ of pulse amplitude, $2 \mathrm{mV}$ of step potential and a potential scan between $-0.6 \mathrm{~V}$ and $0.3 \mathrm{~V}$ at $5 \mathrm{mV} \mathrm{s}^{-1}$. The electrodes obtained after hybridization were denoted as

Ec2c/Ec1SH/AuNPs/MPTS/Au,

Ec2m/Ec1SH/AuNPs/MPTS/Au, $\mathrm{Ec} 2 \mathrm{nc} / \mathrm{Ec} 1 \mathrm{SH} / \mathrm{AuNPs} / \mathrm{MPTS} / \mathrm{Au}, \mathrm{Ec} 2 \mathrm{c}+\mathrm{Lis} / \mathrm{Ec} 1 \mathrm{SH} / \mathrm{AuNPs} / \mathrm{MPTS} / \mathrm{Au}$ for fully complementary (Ec2c), mismatched (Ec2m), non-complementary (Ec2nc) and the mixture of fully complementary E. Coli and Listeria sequences, respectively.

\section{Results and discussion}

\section{Biosensor development: morphological characterization and optimization}

The different steps involved in the preparation of the biosensing organic-inorganic hybrid material were optimized. The first step of the biosensor fabrication consists of generating the polymeric network by sol-gel technology, in which all the components integrating the biosensor will be encapsulated. Since the final properties of the resulting three-dimensional matrix depend strongly on the composition of the sol-gel solution, several volume ratios of MPTS: $\mathrm{H}_{2} \mathrm{O}: \mathrm{CH}_{3} \mathrm{OH}: \mathrm{HCl}$ were tested. From this study, it was concluded that the optimal volume ratio to obtain a homogeneous and transparent solution, without phase separation, after ultrasonic agitation, was 1:3:3:1. The resulting sol-gel solution was stored for two hours at room temperature. During this time, the hydrolysis and polycondensation reactions, responsible for the polymeric network generation, started. Several settle times, from 1 to 6 hours, were assayed in order to ascertain their influence in the resulting biosensor response. An optimal time of 2 hours was selected because for this time the biosensor reaches the maximum response. Likewise, the optimal contact time between the as-prepared sol-gel solution and the gold surface was selected. In this case, the maximum biosensor response corresponded to a contact time of 15 minutes. During this process, the 3D polymeric network was anchored onto gold surface through thiol tail groups, which have also served to incorporate the AuNPs. 
We have assessed by Scanning Electron Microscopy (SEM) the incorporation of gold nanoparticles into the MPTS network. Since it is not possible with surface techniques to check whether gold nanoparticles have been in fact incorporated inside the MPTS network, we devised the following experiment by SEM. We formed on purpose a thick deposit of the MPTS/AuNPs preparation onto the gold surface with a relatively low initial concentration of AuNPs. In the corresponding SEM image, the MPTS regions yielded a dark contrast, while the AuNPs gave a bright contrast due to their metallic character (figure 1A). Note that the nanoparticles tend to form relative large aggregates. However, the brightness of the nanoparticles varied from very intense to rather low, as can be observed in figure 1B that was recorded at a landing electron energy of $6 \mathrm{keV}$. We interpreted this contrast range as due to the location of the nanoparticles inside the MPTS network. Thus, those appearing brighter were located close to the surface. In contrast, those displaying a low contrast were buried to some extent inside this network, which implied that less low energetic secondary electrons were emitted by them and, accordingly, less could come through the network layer above them. We have further checked this interpretation by imaging the same area but at a slightly higher landing energy, $8 \mathrm{keV}$ (figure 1C).
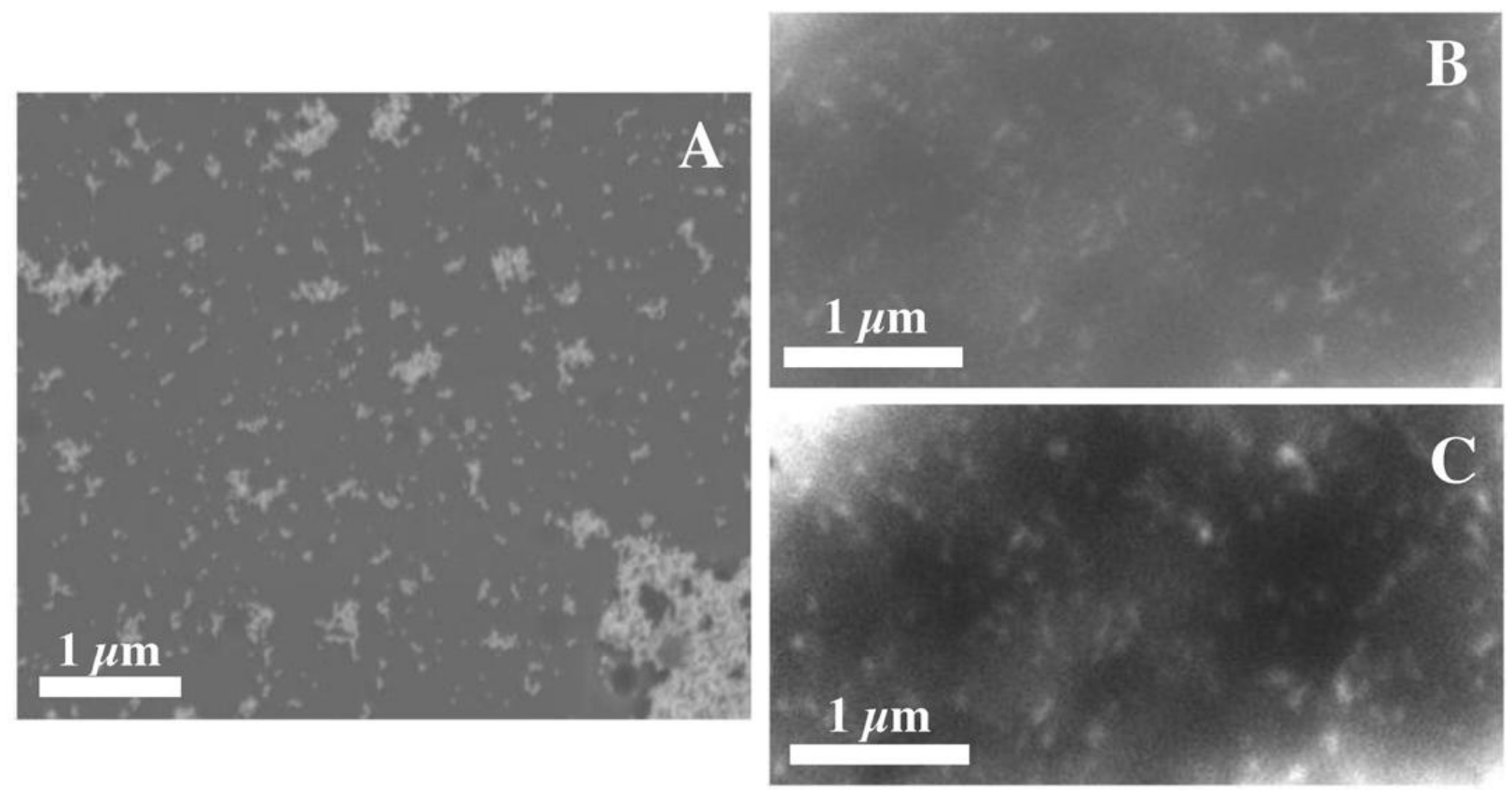

Figure 1. SEM images taken on a relatively thick deposit of AuNPs/MPTS/Au: (A) Large image showing the bright AuNPs and dark MPTS region. A same area displaying both structures imaged at $6 \mathrm{keV}(\mathrm{B})$ and $8 \mathrm{keV}$ (C) electron landing energy. Note how more brighter spots are detected at $8 \mathrm{keV}$. 
Under this condition, the brightness of the formerly low contrasted nanoparticles increases because more secondary electrons are generated at larger depths and, consequently, a larger amount of them are able to escape and be collected [38] leading to brighter contrast. Therefore, this qualitative experiment supports the incorporation of the AuNPs into the MPTS network. Recent reports [39] from other authors also confirm that AuNPs are integrated in 3D polymeric networks containing thiolated groups. Furthermore, we have analyzed by AFM at higher resolution those areas where the nanoparticles form aggregates at the surface (figure 2A). Indeed, this image shows rounded and smooth structures with sizes consistent with those of the gold nanoparticles.
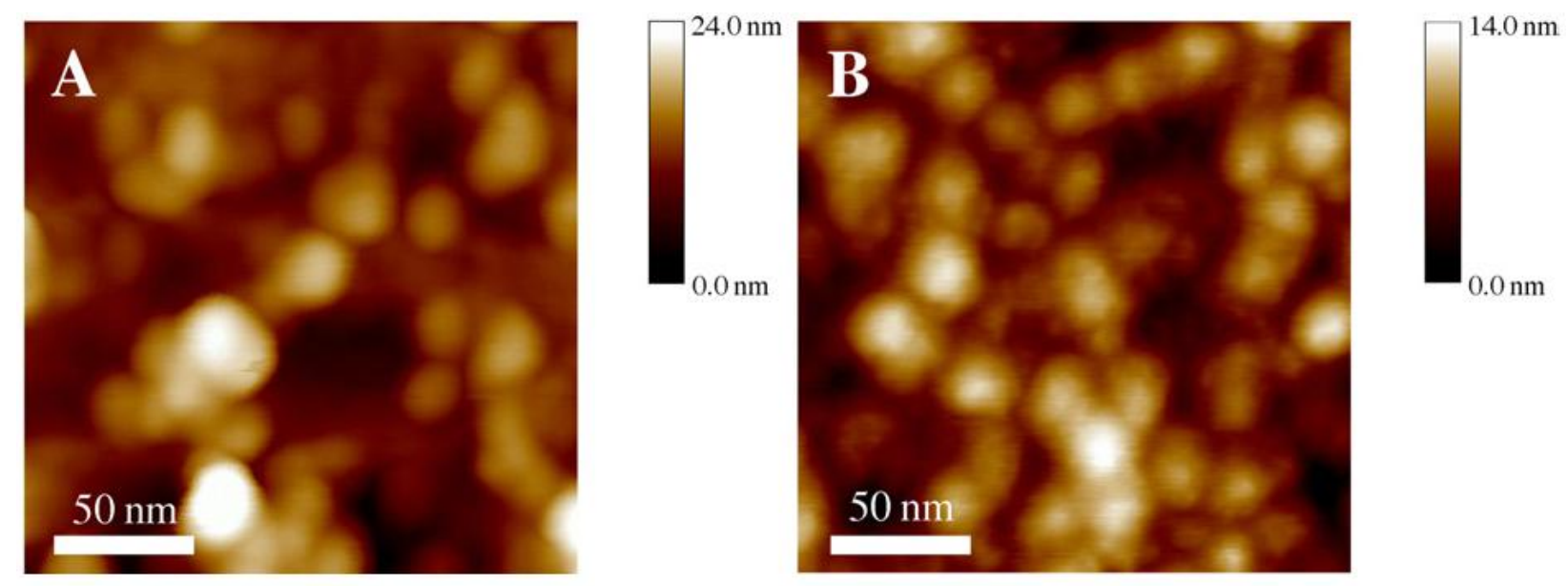

Figure 2: $200 \times 200 \mathrm{~nm}^{2}$ AFM images of the AuNPS aggregates formed on the surface of the (A) AuNPs/MPTS/Au and (B) Ec1SH/AuNPs/MPTS/Au systems.

In order to obtain the final Ec1SH/AuNPs/MPTS/Au electrode, different amounts of thiolated capture probe (Ec1SH) were tested. In the optimal conditions, modified electrodes were obtained by transferring $10 \mu \mathrm{L}$ of $100 \mu \mathrm{M}$ HS-ssDNA (Ec1SH) solution onto the surface of AuNPs/MPTS/Au electrode; this means a total amount of $1 \mathrm{nmol}$ of Ec1SH. In figure 2B is shown a higher resolution AFM image of the biosensor surface, in particular a region where the gold nanoparticles aggregate. Clearly, the AuNPS are observed but, interestingly, small nanostructures (0.5-1 nm high) are scattered on their surface. Since these nanostructures were not observed in figure 2A and their dimensions are consistent with previous results [40] for a similar system, they can be related to the capture probe (Ec1SH). It should be noted that AFM only measure the exposed surface of the nanoparticles. 
In the detection step, $\left[\mathrm{Ru}\left(\mathrm{NH}_{3}\right)_{5} \mathrm{~L}\right]^{2+}$ complex was electrochemically incorporated on the double stranded DNA (ds-DNA) formed onto the electrode surface after hybridization. Different concentrations of $\left[\mathrm{Ru}\left(\mathrm{NH}_{3}\right)_{5} \mathrm{~L}\right]^{2+}$ complex (up to $500 \mu \mathrm{M}$ ) and of supporting electrolyte (from 0.05 to $2 \mathrm{M}$ ) were tested in order to obtain the optimal electrochemical response. Best analytical signals were obtained using a concentration of $\left[\mathrm{Ru}\left(\mathrm{NH}_{3}\right)_{5} \mathrm{~L}\right]^{2+}$ of $100 \mu \mathrm{M}$ in the $\mathrm{CV}$ cycling process and $1 \mathrm{M} \mathrm{KNO}_{3}$ was employed as supporting electrolyte in the DPV measurements. In table 1 are summarized the optimal conditions for the preparation of the biosensor and for the detection of the hybridization event.

\begin{tabular}{lll} 
Table $\mathbf{1}$ \\
Optimal conditions for both biosensor development and detection of hybridization event. \\
\hline Biosensor development & & \\
\hline MPTS: $\mathrm{H}_{2} \mathrm{O}: \mathrm{CH}_{3} \mathrm{OH}: \mathrm{HCl}$ volume ratio & $1: 3: 3: 1$ & Detection of hybridization event \\
Settle time & $2 \mathrm{~h}$ & $\left.\mathrm{Ru}_{(\mathrm{NH}}\right)_{5} \mathrm{~L}$ concentration \\
Contact time & $15 \mathrm{~min}$ & Supporting electrolyte \\
HS-ssDNA (Ec1SH) probe amount & $1 \mathrm{nmol}$ & \\
\hline
\end{tabular}

\section{Ec1SH/AuNPs/MPTS/Au biosensor response}

Gold screen-printed electrodes modified with thiolated DNA probe (Ec1SH/AuNPs/MPTS/Au) were incubated in solutions containing the target (Ec2c) ss-DNA, as mentioned in the procedure section, to achieve hybridization. Hybridization was detected by changes in the electroactivity of the metal redox center within the $\left[\mathrm{Ru}\left(\mathrm{NH}_{3}\right)_{5} \mathrm{~L}\right]^{2+}$ complex. Then, the $\left[\mathrm{Ru}\left(\mathrm{NH}_{3}\right)_{5} \mathrm{~L}\right]^{2+}$ complex was placed on the surface, cycling the potential from -0.5 to $0.1 \mathrm{~V}$ at $100 \mathrm{mV} \mathrm{s}^{-1} 150$ times. Afterwards, DPVs were recorded. Figure 3 shows the differential pulse voltammograms for Ec1SH/AuNPs/MPTS/Au before (curve A) and after (curve B) hybridization with a $30 \mu \mathrm{M}$ complementary target (Ec2c) solution. As can be seen, a well-defined wave is observed around $-0.23 \mathrm{~V}$, which is very convenient as a mediating system since it avoid side reactions. The analytical signal is significantly higher when the hybridization event has taken place (curve B) due to $\left[\mathrm{Ru}\left(\mathrm{NH}_{3}\right)_{5} \mathrm{~L}\right]^{2+}$ complex binds to double-stranded DNA through intercalation more efficiently than to single stranded DNA. It also corroborates that the threedimensional polymeric network allows to develop bioanalytical platforms that provide a biocompatible environment that preserves the original recognition capability of ss-DNA probe. In addition, the porous material obtained lets to the ss-DNA target to reach the ss-DNA probe through their accessible pores. 


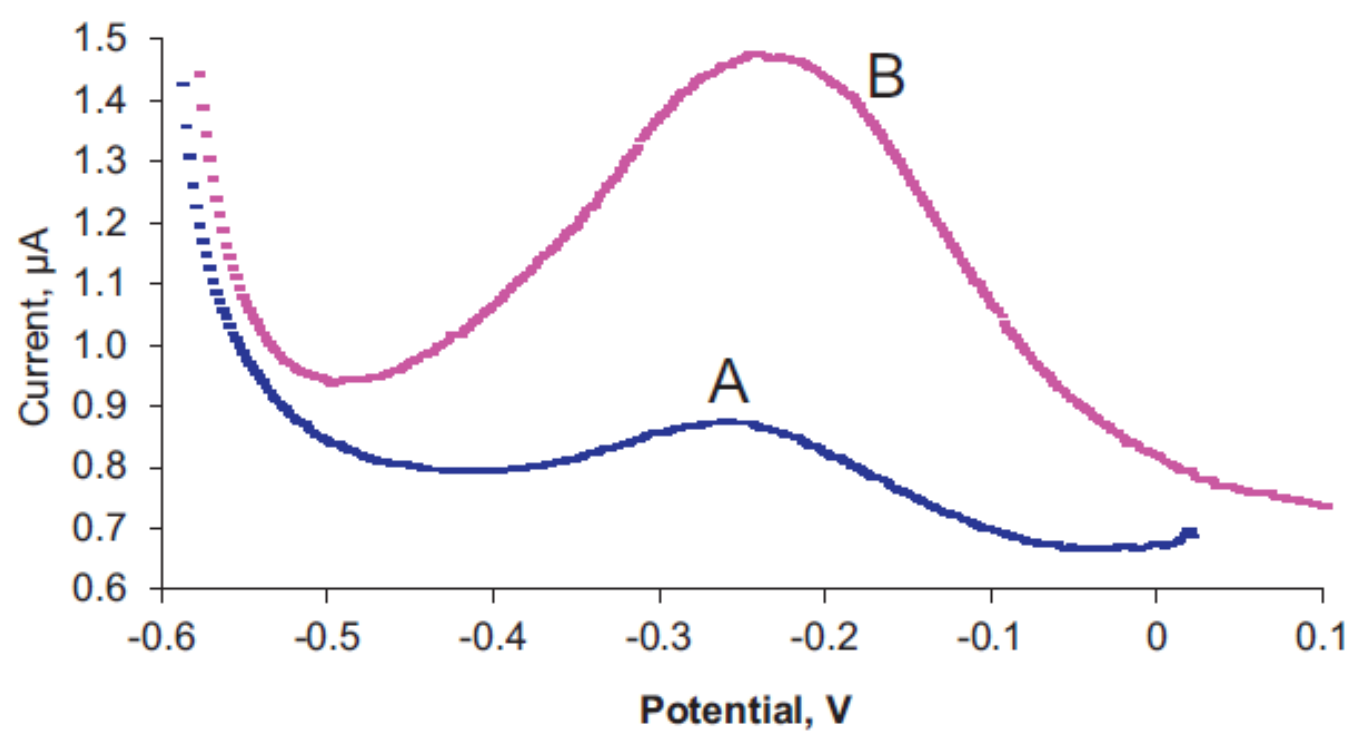

Figure 3. Differential pulse voltammograms in $1 \mathrm{M} \mathrm{KNO}_{3}$ for Ec1SH/AuNPs/MPTS/Au (A) before hybridization and (B) after hybridization with Ec2c. Other conditions are: $\left[\mathrm{Ru}\left(\mathrm{NH}_{3}\right)_{5} \mathrm{~L}\right]=$ $100 \mu \mathrm{M}$. [Ec2c] $=30 \mu \mathrm{M}$. Scan rate: $5 \mathrm{mVs}^{-1}$, pulse amplitude: $50 \mathrm{mV}$, step potential: $2 \mathrm{mV}$.

The developed biosensor Ec1SH/AuNPs/MPTS/Au was also incubated in solutions containing as target: a fully complementary (Ec2c), a non-complementary (Ec2nc) or a mismatched sequence $(\mathrm{Ec} 2 \mathrm{~m})$. Figure 4 shows results obtained for a target solution concentration of $100 \mu \mathrm{M}$.

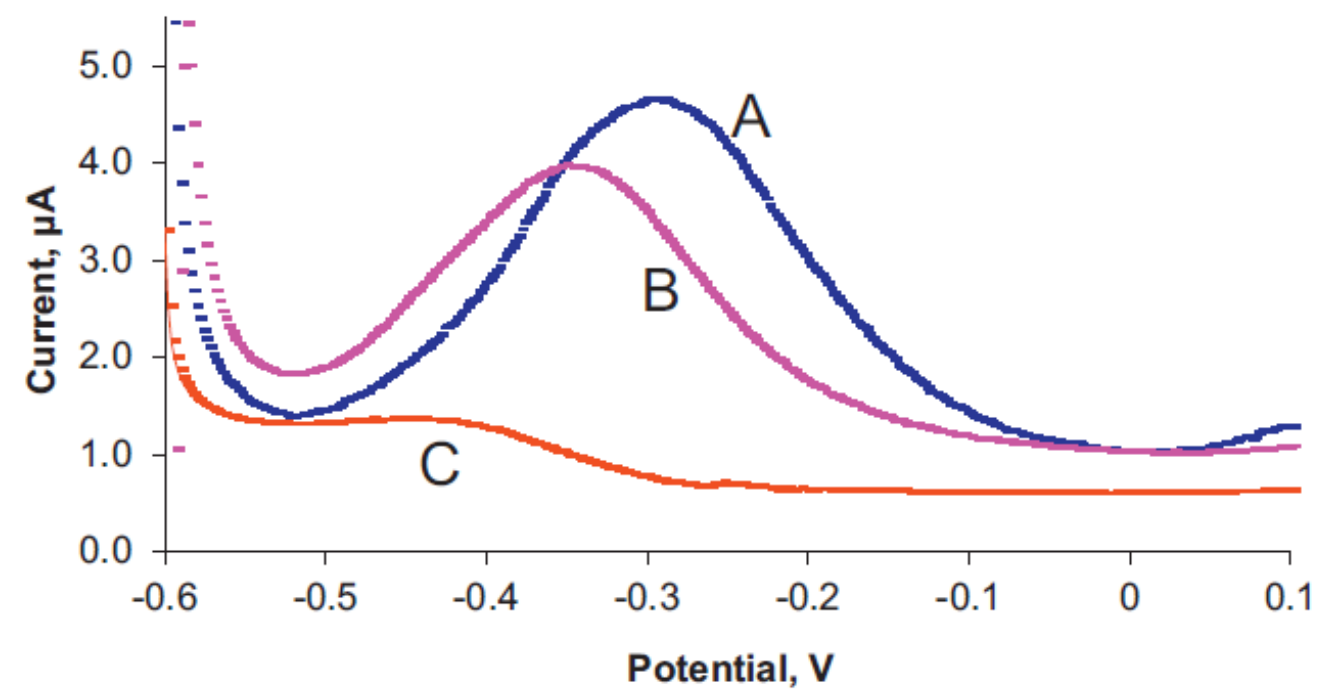

Figure 4. Differential pulse voltammograms in $1 \mathrm{M} \mathrm{KNO}_{3}$ for Ec1SH/AuNPs/MPTS/Au after hybridization with (A) a complementary sequence (Ec2c), (B) a mismatched sequence (Ec2m) and $(C)$ a non-complementary sequences $(\mathrm{Ec} 2 \mathrm{nc})$. $[\mathrm{Ec} 2 \mathrm{c}]=[\mathrm{Ec} 2 \mathrm{~m}]=[\mathrm{Ec} 2 \mathrm{nc}]=100 \mu \mathrm{M}$. Other conditions as in Figure 3. 
In the case of non-complementary sequence (curve C), the current intensity obtained was very low due to the absence of hybridization. In the presence of one mismatch in the middle of the ss-DNA sequence (Ec2m) (curve B), an important current intensity was observed but lower than in the case of the complementary one (curve A). Furthermore, it can be observed (figure 4) that when hybridization has taken place with a mismatched strand (curve B), a displacement of peak potential to more negative values occurs in comparison with complementary strand (curve A). This displacement is due to non-specific interactions, probably in an electrostatic mode, between the probe strand and non- complementary sequences, being higher when the number of electrostatic interactions increases as is the case of the non-complementary strand (curve C).

Figure 5 shows bare diagrams of the normalized current response for $100 \mu \mathrm{M}$ of several targets, including the as-mentioned (a fully complementary, a non-complementary or a mismatched sequence). In this figure, we have represented (I-Io/Io) values where $I o$ and $I$ correspond to the current intensity before and after hybridization, respectively, in order to avoid electrode-to-electrode variations and to compare the peak current intensities obtained for each target. As mentioned above, hybridization with a non-complementary target yields a non significant increment in the current, indicating that the nonspecific adsorption was small. Conversely, hybridization with complementary target gives rise to a remarkable increase. The presence of a mismatch in the target can be detected.

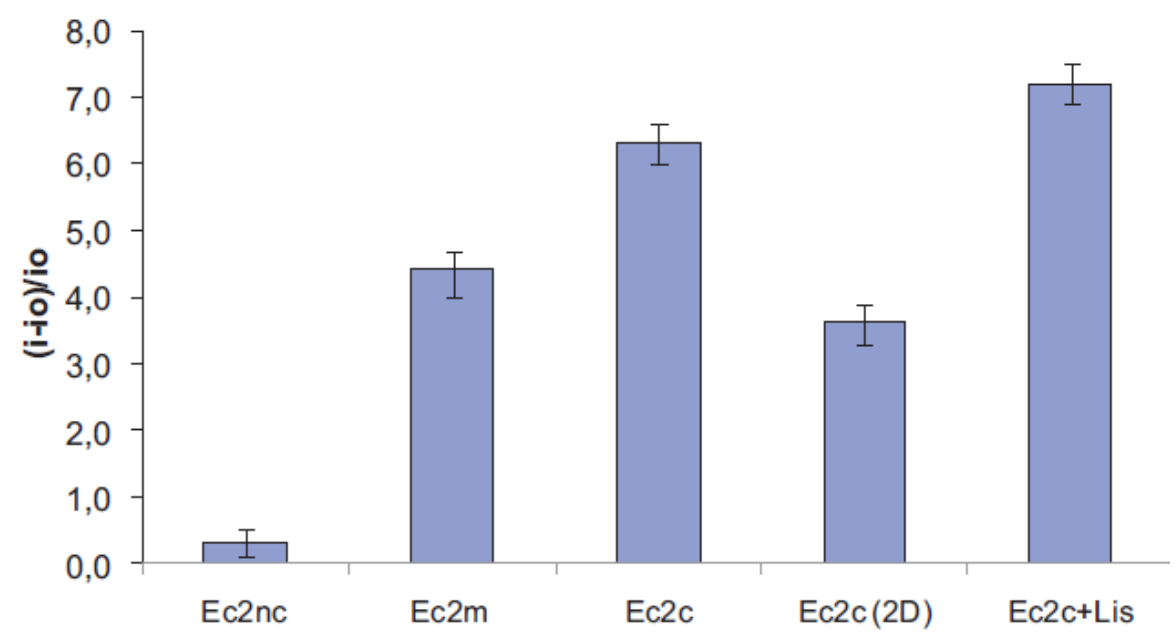

Figure 5. Bar diagram of the biosensor response versus non-complementary (Ec2nc), mismatched (Ec2m) and complementary (Ec2c) E. Coli sequences and a mixture of a complementary (Ec2c) E. Coli sequence and a Listeria sequence (Ec2c+Lis); and for Ec1SH/Au 
platform after hybridization versus a complementary (Ec2c) E. Coli sequence. Conditions are: $\left[\mathrm{Ru}\left(\mathrm{NH}_{3}\right)_{5} \mathrm{~L}\right]=100 \mu \mathrm{M}$, concentrations of different sequences $=100 \mu \mathrm{M}$, scan rate: $5 \mathrm{mVs}^{-1}$, pulse amplitude: $50 \mathrm{mV}$, step potential: $2 \mathrm{mV}$.

Moreover, the current response for a biosensor based on a gold screen-printed electrode modified with the thiolated DNA probe (Ec1SH/Au) towards the fully complementary (Ec2c) sequence has been obtained and it is presented in figure 5. As can be observed, the response, denoted as Ec2c (2D), is lower than that obtained for the Ec1SH/AuNPs/MPTS/Au platform. Furthermore, we have studied the response of the Ec1SH/MPTS/Au platform towards the fully complementary (Ec2c) sequence (data not shown). A remarkably low current response has been obtained probably due to the kinetic barrier of the self-assembled silicate network to the electron transfer [33].These results show that the presence of the AuNPs becomes essential in order to enhance the biosensor response. The role of the nanoparticles is twofold. On one hand, gold nanoparticles can be incorporated both inside and on the surface of a MPTS based three dimensional sol-gel network [32]. Thus, a MPTS/Au surface allows immobilizing a higher amount of gold nanoparticles than a similar one but without the three dimensional network. Likewise, due to the typical nanoporosity of 3D sol-gel networks, the DNA molecules can penetrate into the polymeric network, reaching those AuNPs buried into the MPTS. Therefore, in this system the DNA probe loading is enhanced. On the other hand, AuNPs act also as tiny conduction centers, facilitating the electron transfer between the redox indicator and the transducer [33]. This combination of properties leads to an improvement of the sensitivity of the resulting biosensor.

Finally, taking into account the possibility of the presence of interferents in real E. Coli samples, we have studied the biosensor response in samples containing the analyte and a possible interferent as Listeria in the same concentration $(100 \mu \mathrm{M})$. As can be observed in figure 5, in the presence of this interferent the response is $12 \%$ higher than that obtained in its absence. Therefore, this result suggests that the biosensor can be successfully employed to detect $E$. Coli sequences even in the presence of a possible interferent substance.

\section{Ec1SH/AuNPs/MPTS/Au biosensor analytical properties}

The current response of the Ec1SH/AuNPs/MPTS/Au biosensor increases with the amount of the target ss-DNA used, as can be observed in figure S1. The normalized response (I-Io/Io) 
versus the Ec2c concentration shows a good correlation $\left(y=0.11 x+0.14, r^{2}=0.995\right)$ over the range from 2.1 to $40 \mu \mathrm{M}$ (from 21 to $400 \mathrm{pmol}$ ). The detection limit, calculated as the ratio between three times the standard deviation of the Ec1SH/AuNPs/MPTS/Au intensity current (before hybridization) and the sensitivity, was $0.63 \mu \mathrm{M}(6.3 \mathrm{pmol})$. The quantification limit, calculated as the ratio between ten times the standard deviation of the Ec1SH/AuNPs/MPTS/Au intensity current (before hybridization) and the sensitivity, was $2.1 \mu \mathrm{M}(21 \mathrm{pmol})$. The reproducibility using four different biosensors, expressed as the relative standard deviation (RSD), was $4.3 \%$ for a concentration of complementary sequence $(\mathrm{Ec} 2 \mathrm{c})$ of $10 \mu \mathrm{M}$. In order to assess the storage stability of the resulting biosensor, a set of 30 electrodes were modified at the same time and kept at $4^{\circ} \mathrm{C}$ after modification. The magnitude of the current response for $10 \mu \mathrm{M}$ Ec2c was monitored every day with a different electrode during one month, obtaining that after 25 days, the biosensor remained active. The RSD calculated was $6.5 \%$.

These results can be compared with those derived from several previous experimental works that employ similar biosensing platforms for detecting sequences from bacterium specimens, but with a 2D configuration (i.e, without a 3D sol-gel network). In all cases, the DNA capture probes were 25-mer thiolated synthetic oligonucleotides sequences and the transducers were gold electrodes. Furthermore, the detection of hybridization event was achieved using the same $\left[\mathrm{Ru}\left(\mathrm{NH}_{3}\right)_{5} \mathrm{~L}\right]^{2+}$ complex. The comparison is presented in table 2. As can be seen in this table, for the biosensor developed by immobilization of the probe onto bare gold electrodes (DNA/Au), a detection limit of 92 pmol was found [35]. In a second approach [8], AuNPs were electrodeposited onto the gold electrode surface (DNA/AuNPs/Au) prior to the immobilization of the capture probe. In this case, a better detection limit is achieved ( $25 \mathrm{pmol})$, as can be expected due to the increase of the specific surface because of the presence of AuNPs. For the biosensor developed in the present work, the main particularity is the presence of both AuNPs and a 3D sol-gel network. In this case, the specific surface is also increased due to the inclusion of the AuNPs but in a higher amount due to their incorporation in the 3D network. Moreover, these AuNPs also contribute to enhance the electron transfer.

\begin{tabular}{|c|c|c|c|c|c|c|}
\hline Reference & Biosensing platform & Analyte & Linear concentration range ( $\mathrm{pmol})$ & $\mathrm{DL}(\mathrm{pmol})$ & Reproducibility & Stability (days) ${ }^{\mathrm{a}}$ \\
\hline [35] & $\mathrm{DNA} / \mathrm{Au}$ & 25 mer H. pylori & $106-708(r=0.995)$ & 92 & $94 \%$ & n.r. \\
\hline [8] & DNA/AuNPs/Au & 25 mer H. pylori & $40-800(r=0.998)$ & 25 & $79 \%$ & 1 \\
\hline Present work & DNA/MPTS/AuNPs/Au & 25 mer E. coli & $21-400(r=0.997)$ & 6.3 & $\mathrm{RSD}=4.3 \%(n=4, C=10 \mu \mathrm{M})$ & 25 \\
\hline
\end{tabular}


Then, it can be concluded that the most important differences between the above mentioned biosensors and the present work are the simultaneous presence of a three-dimensional sol-gel network and AuNPs, which allows to measure a lower concentration and to obtain a lower detection limit. In addition, one of the additional advantages that one would expect by using three dimensional arrangements is a long-term stability as can be confirmed after comparison of our data with those obtained on similar biosensors but without MPTS [8].

\section{Conclusions}

The three-dimensional polymeric network obtained by the sol-gel process allows to develop bioanalytical platforms that provide a biocompatible environment preserving the original structure of ss-DNA probe and letting to the ss-DNA target to reach the ss-DNA probe through their accessible pores. $\left[\mathrm{Ru}\left(\mathrm{NH}_{3}\right)_{5} \mathrm{~L}\right]^{2+}$ complex, intercalated into ds-DNA layer formed after hybridization, exhibits a well-defined wave that allows to detect the hybridization event. It has also been possible to distinguish the different strands (complementary, non-complementary and mismatched), from the differences in the peak potential and peak intensity values obtained for each case. The analytical properties of the disposable electrochemical biosensors developed were studied, obtaining a linear response in the range of 21 to 400 pmoles and a detection limit of 6.3 pmoles with a good reproducibility $(\mathrm{RSD}=4.3 \%)$. The presence of both the three dimensional sol-gel networks and the gold nanoaparticles allows the incorporation of high amount of ss-DNA probe improving the performance of the final biosensing device. In particular, it makes possible to obtain a lower detection limit and a higher stability compared with biosensors of similar characteristics. The resulting biosensors are stable under storage conditions at least for 25 days.

\section{Acknowledgements}

This work has been supported by Comunidad Autónoma de Madrid (project No. S2009/PPQ-1642, AVANSENS) and Ministerio de Ciencia e Innovación (project No. CTQ201128157 and FIS2012-38866-C05-05). 


\section{References}

[1] H. Min, N. Sullivan, D. Allara, S. Tadigadapa, Nanoporous Gold: A High Sensitivity and Specificity Biosensing Substrate, Procedia Engineering 25 (2011) 1469-1472.

[2] M. Minunni, S. Tombelli, R. Scielzi, I. Mannelli, M. Mascini, C. Gaudiano, Detection of $\beta$-thalassemia by a DNA piezoelectric biosensor coupled with polymerase chain reaction, Anal. Chim. Acta 481 (2003) 55-64.

[3] G. Chang, J. Hu, W. Lu, X. Qin, A.M. Asiri, A.O. Al-Youbi, X. Sun, Carbon Nanocapsules as an Effective Sensing Platform for Fluorescence-Enhanced Nucleic Acid Detection, J. Nanosci. Nanotechno. 12 (2012) 3775-3780.

[4] E.V. Dmitrienko, E.A. Khomyakova, I.A. Pyshnaya, A.G. Bragin, V.E. Vedernikov, D.V. Pyshnyi, Oligonucleotide Derivatives in the Hybridization Analysis of Nucleic Acids: II. Isothermal Signal Amplification in DNA Analysis by Minisequencing, Russ. J. Bioorg. Chem. 36 (2010) 734-745.

[5] R. Wang, M. Minunni, S. Tombelli, M. Mascini, A new approach for the detection of DNA sequences in amplified nucleic acids by a surface plasmon resonance biosensor, Biosens. Bioelectron. 20 (2004) 598-605.

[6] K. Kleo, A. Kapp, L. Ascher, F. Lisdat, Detection of vaccinia virus DNA by quartz crystal microbalance, Anal. Biochem. 418 (2011) 260-266.

[7] A. Abbaspour, L. Baramakeh, S.M. Nabavizadeh, Development of a disposable sensor for electrocatalytic detection of guanine and ss-DNA using a modified sol-gel screenprinted carbon electrode, Elctrochim. Acta 52 (2007) 4798-4803.

[8] T. García, E. Casero, M. Revenga-Parra, J. Martin-Benito, F. Pariente, L. Vázquez, E. Lorenzo, Architectures based on the use of gold nanoparticles and ruthenium complexes as a new route to improve genosensor sensitivity, Biosens. Bioelectron. 24 (2008) 184190.

[9] M.V. Pozo, C. Alonso, F. Pariente, E. Lorenzo, DNA Biosensor for Detection of Helicobacter pylori Using Phen-dione as the Electrochemically Active Ligand in Osmium Complexes, Anal. Chem. 77 (2005) 2550-2557.

[10] A.A. Ansari, R. Singh, G. Sumana, B.D. Malhotra, Sol-gel derived nano-structured zinc oxide film for sexually transmitted disease sensor, Analyst 134 (2009) 997-1002. 
[11] Y. Fu, R. Yuan, L. Xu, Y. Chai, Y. Liu, D. Tang, Y. Zhang, Electrochemical impedance behavior of DNA biosensor based on colloidal Ag and bilayer two-dimensional sol-gel as matrices, J. Biochem. Biophys. Methods 62 (2005) 163-174.

[12] F. Li, W. Chen, S. Zhang, Development of DNA electrochemical biosensor based on covalent immobilization of probe DNA by direct coupling of sol-gel and self-assembly technologies, Biosens. Bioelectron. 24 (2008) 781-786.

[13] S. Mohan, P. Srivastava, S.N. Maheshwari, S. Sundar, R. Prakash, Nano-structured nickel oxide based DNA biosensor for detection of visceral leishmaniasis (Kala-azar), Analyst 136 (2011) 2845-2851.

[14] P.R. Solanki, A. Kaushik, P.M. Chavhan, S.N. Maheshwari, B.D. Malhotra, Nanostructured zirconium oxide based genosensor for Escherichia coli detection, Electrochem. Commun. 11 (2009) 2272-2277.

[15] M. Barbadillo, E. Casero, M.D. Petit-Domínguez, L. Vázquez, F. Pariente, E. Lorenzo, Gold nanoparticles-induced enhancement of the analytical response of an electrochemical biosensor based on an organic-inorganic hybrid composite material, Talanta 80 (2009) 797-802.

[16] M. Barbadillo, E. Casero, M.D. Petit-Domínguez, F. Pariente, E. Lorenzo, L. Vázquez, Surface study of the building steps of enzymatic sol-gel biosensors at the micro- and nano-scales, J. Sol-Gel Sci. Techn. 58 (2011) 452-462.

[17] G. Cabello-Carramolino, M.D. Petit-Dominguez, Application of new sol-gel electrochemical sensors to the determination of trace mercury, Anal. Chim. Acta 614 (2008) 103-111.

[18] G. Cabello-Carramolino, M.D. Petit-Dominguez, Development of new sol-gel carbon composite electrodes and their application as electrochemical sensors, Microchim. Acta 164 (2009) 405-410.

[19] A.M. Parra-Alfambra, E. Casero, M.D. Petit-Dominguez, M. Barbadillo, F. Pariente, L. Vazquez, E. Lorenzo, New nanostructured electrochemical biosensors based on threedimensional (3-mercaptopropyl)-trimethoxysilane network, Analyst 136 (2011) 340-347.

[20] J.A. Cox, P.M. Hensley, C.L. Loch, Evaluation of Polycation-Stabilized Lactate Oxidase in a Silica Sol-Gel as a Biosensor Platform, Microchim. Acta 142 (2003) 1-5. 
[21] A. Kumar, R.R. Pandey, B. Brantley, Tetraethylorthosilicate film modified with protein to fabricate cholesterol biosensor, Talanta 69 (2006) 700-705.

[22] C.I. Li, Y.H. Lin, C.L. Shih, J.P. Tsaur, L.K. Chau, Sol-gel encapsulation of lactate dehydrogenase for optical sensing of L-lactate, Biosens. Bioelectron. 17 (2002) 323-330.

[23] A. Salimi, R.G. Compton, R. Hallaj, Glucose biosensor prepared by glucose oxidase encapsulated sol-gel and carbon-nanotube-modified basal plane pyrolytic graphite electrode, Anal. Biochem. 333 (2004) 49-56.

[24] S. Singh, R. Singhal, B.D. Malhotra, Immobilization of cholesterol esterase and cholesterol oxidase onto sol-gel films for application to cholesterol biosensor, Anal. Chim. Acta 582 (2007) 335-343.

[25] E.I. Yashina, A.V. Borisova, E.E. Karyakina, O.I. Shchegolikhina, M.Y. Vagin, D.A. Sakharov, A.G. Tonevitsky, A.A. Karyakin, Sol-Gel Immobilization of Lactate Oxidase from Organic Solvent: Toward the Advanced Lactate Biosensor, Anal. Chem. 82 (2010) 1601-1604.

[26] A.A. Ansari, A. Kaushik, P.R. Solanki, B.D. Malhotra, Sol-gel derived nanoporous cerium oxide film for application to cholesterol biosensor, Electrochem. Commun. 10 (2008) 1246-1249.

[27] X. Chen, S. Dong, Sol-gel-derived titanium oxide/copolymer composite based glucose biosensor, Biosens. Bioelectron. 18 (2003) 999-1004.

[28] E. Topoglidis, Y. Astuti, R. Duriaux, M. Grätzel, J.R. Durrant, Direct Electrochemistry and Nitric Oxide Interaction of Heme Proteins Adsorbed on Nanocrystalline Tin Oxide Electrodes, Langmuir 19 (2003) 6894-6900.

[29] B.K. Jena, C.R. Raj, Electrochemical Biosensor Based on Integrated Assembly of Dehydrogenase Enzymes and Gold Nanoparticles, Anal. Chem. 78 (2006) 6332-6339.

[30] B.K. Jena, C.R. Raj, Amperometric L-Lactate Biosensor Based on Gold Nanoparticles, Electroanalysis 19 (2007) 816-822.

[31] H. Chen, S. Dong, Direct electrochemistry and electrocatalysis of horseradish peroxidase immobilized in sol-gel-derived ceramic-carbon nanotube nanocomposite film, Biosens. Bioelectron. 22 (2007) 1811-1815. 
[32] J. Jia, B. Wang, A. Wu, G. Cheng, Z. Li, S. Dong, A method to construct a thirdgeneration horseradish peroxidase biosensor: self-assembling gold nanoparticles to threedimensional sol-gel network, Anal. Chem. 74 (2002) 2217-2223.

[33] S. Barathi, N. Nogami, S. Ikeda, Novel electrochemical interface with a tunable kinetic barrier by self-assembling organically modified silica gel and gold nanoparticles, Langmuir 17 (2001) 1-3.

[34] T. García, E. Casero, M. Revenga-Parra, E. Pariente, E. Lorenzo, Dual-Stage DNA Sensing: Recognition and Detection, Anal. Chem. 80 (2008) 9443-9449.

[35] T. García, M. Revenga-Parra, H.D. Abruña, F. Pariente, E. Lorenzo, Single-Mismatch Position-Sensitive Detection of DNA Based on a Bifunctional Ruthenium Complex, Anal. Chem. 80 (2008) 77-84.

[36] T. García, M.G. Fernández-Barrena, M. Revenga-Parra, A. Nuñez, E. Casero, F. Pariente, J. Prieto, E. Lorenzo, Disposable sensors for rapid screening of mutated genes, Anal. Bioanal. Chem. 398 (2010) 1385-1393.

[37] C.G. Kuehn, H. Taube, Ammineruthenium complexes of hydrogen sulfide and related sulfur ligands, J. Am. Chem. Soc. 98 (1976) 689-702.

[38] A. Shih, J. Yater, C. Hor, R. Abrams, Secondary electron emission studies, Appl. Surf. Sci. 111 (1997) 251-258.

[39] R. Ouyang, S.A. Bragg, J.Q. Chambers, Z.L. Xue, Flower-like self-assembly of gold nanoparticles for highly sensitive electrochemical detection of chromium(VI), Anal. Chim. Acta 722 (2012) 1-7.

[40] E. Casero, M. Darder, D.J. Diaz, F. Pariente, J.A. Martin-Gago, H. Abruña, E. Lorenzo, XPS and AFM Characterization of Oligonucleotides Immobilized on Gold Substrates, Langmuir 19 (2003) 6230-6235.

\section{SUPPLEMENTAL FIGURES}

Figure S1. Calibration curve obtained by plotting the normalized current obtained for Ec1SH/AuNPs/MPTS/Au platform versus the amount of complementary sequence (Ec2c). 